\title{
COPLANAR CONTROL OF A SATELLITE AROUND THE EARTH*
}

\author{
Jean-Baptiste Caillau ${ }^{1}$ and Joseph Noailles ${ }^{1}$
}

\begin{abstract}
We investigate the minimum time transfer of a satellite around the Earth. Using an optimal control model, we study the controllability of the system and propose a geometrical analysis of the optimal command structure. Furthermore, in order to solve the problem numerically, a new parametric technique is introduced for which convergence properties are established.
\end{abstract}

Résumé. On s'intéresse au transfert en temps minimal d'un satellite autour de la Terre. Sur la base d'une modélisation contrôle optimal, on étudie la controlabilité du système et on propose une analyse de la structure de la commande optimale. En outre, afin de procéder à la résolution numérique du problème, une nouvelle méthode paramétrique dont on établit des propriétés de convergence est définie.

Mathematics Subject Classification. 70Q05, 49M30, 93B29.

Received April 25, 2000. Revised December 9, 2000.

\section{INTRODUCTION}

This paper is devoted to the study of the transfer of a satellite around the Earth $[5,7,9,14,15]$. Starting from a low initial and very eccentric orbit, we aim at reaching a geostationnary terminal one. The transfer is assumed to be coplanar (in practice, one should also take into account the inclination), and we control the thrust of the engine in the transfer plane. Since we consider electro-ionic propulsion, the maximum thrust available is very low (v.g. 0.3 Newton). The state of the spacecraft is, for instance, the couple position-speed, though we shall see that the equinoctial elements [18] are better suited both from theoretical and numerical point of view. Contrary to $[5,15]$, the state also comprises the mass of the satellite which is varied during the transfer because of the fuel consumption. Choosing this more realistic model has consequences on controllability, as well as on the structure of the control.

The paper is organised as follows: the optimal control formulation of the transfer is detailed in Section 1. In particular, the two main systems of coordinates on the underlying manifold are described. In Section 2, controllability of the system is investigated, from where the existence of an optimal control proceeds. Then, the structure of the optimal controls, that is the characteristics of the switching points, is studied in Section 3 in a geometric control setting. In Section 4, a new parametric approach for time optimal control problems is presented, the idea being to treat the performance index as a parameter. We focus on convergence properties of

Keywords and phrases: Celestial mechanics, minimum time problems, geometric control, parametric optimal control.

* Supported in part by the French Space Agency (CNES-ENSEEIHT contract 871/94/CNES/1454) and the French Ministry for Education, Higher Education and Research (grant 20INP96).

1 ENSEEIHT-IRIT, UMR 5505 du CNRS, 2 rue Camichel, 31071 Toulouse, France;

e-mail: caillau@enseeiht.fr \& jnoaille@enseeiht.fr 
the associated algorithm under quite general assumptions. Finally, we use this technique to solve numerically the transfer problem in Section 5. The results are analysed in the light of Section 3 statements.

\section{Problem statement}

We first describe the dynamics of the system. It involves the state $x$ of the satellite, its mass $m$, and the thrust $u$ of the engine:

$$
\begin{aligned}
\dot{x} & =f_{0}(x)+1 / m\left(u_{1} f_{1}(x)+u_{2} f_{2}(x)\right) \\
\dot{m} & =-\delta|u|
\end{aligned}
$$

where the drift $f_{0}$ represents the Keplerian action of the Earth. The norm |.| in (1.2) is Euclidean (the variation of the mass is proportional to the fuel consumption; when $\delta=0$, we have a constant mass model). The same convention that finite-dimensional norms |.| are 2-norms will be used throughout the paper. The canonical Euclidean dot product will be denoted by (.|.), and the transpose of a linear operator $A$ by ${ }^{t} A$. Equation (1.1) makes sense for $m>0$ on the smooth ${ }^{1}$ open submanifold $M^{4}$ of $\mathbf{R}^{4}$ (2D transfer) defined in cartesian coordinates $\left(r_{1}, r_{2}, v_{1}, v_{2}\right)$ by:

$$
|r|>0,|v|<\sqrt{2 \mu^{0} /|r|} .
$$

Definition (1.3) ensures that the trajectories remain elliptic (the aforementioned $\mu^{0}$ is the gravitation constant of the Earth). In cartesian coordinates, the dynamics writes $\dot{r}=v, \dot{v}=-\mu^{0} r /|r|^{3}+u / m$ so that

$$
\begin{aligned}
& f_{0}=v \partial_{r}-\mu^{0} r /|r|^{3} \partial_{v} \\
& f_{1}=\partial_{v_{1}}, f_{2}=\partial_{v_{2}} .
\end{aligned}
$$

Another set of coordinates is also available on $M^{4}$, namely the orbital parameters $\left(P, e_{x}, e_{y}, L\right)$ that define the ellipse osculating to the trajectory (and the position of the spacecraft on it, see [18]): $P$ is the semi-latus rectum, $e=\left(e_{x}, e_{y}\right)$ is the eccentricity vector, and $L$ is the $2 \pi$-periodic true longitude $(L=\omega+w \in \mathbf{R} / 2 \pi \mathbf{Z}, \omega$ argument of the perigee, $w$ true anomaly). Of course, $P>0$ and $|e|<1$ on $M^{4}$ because of (1.3). The change of variables formulas are

$$
\begin{aligned}
& r_{1}=P / W \cos L \\
& r_{2}=P / W \sin L \\
& v_{1}=-\sqrt{\mu^{0} / P}\left(e_{y}+\sin L\right) \\
& v_{2}=\sqrt{\mu^{0} / P}\left(e_{x}+\cos L\right)
\end{aligned}
$$

with

$$
W=1+e_{x} \cos L+e_{y} \sin L .
$$

Consequently, in these new coordinates,

$$
\begin{aligned}
& f_{0}=\sqrt{\mu^{0} / P} W^{2} / P \partial_{L} \\
& f_{1}=\sqrt{P / \mu^{0}}\left(\sin L \partial_{e_{x}}-\cos L \partial_{e_{y}}\right) \\
& f_{2}=\sqrt{P / \mu^{0}}\left(2 P / W \partial_{P}+\left(\cos L+\left(e_{x}+\cos L\right) / W\right) \partial_{e_{x}}+\left(\sin L+\left(e_{y}+\sin L\right) / W\right) \partial_{e_{y}}\right)
\end{aligned}
$$

\footnotetext{
${ }^{1}$ As usual, by smooth we mean $C^{\infty}$-smooth.
} 


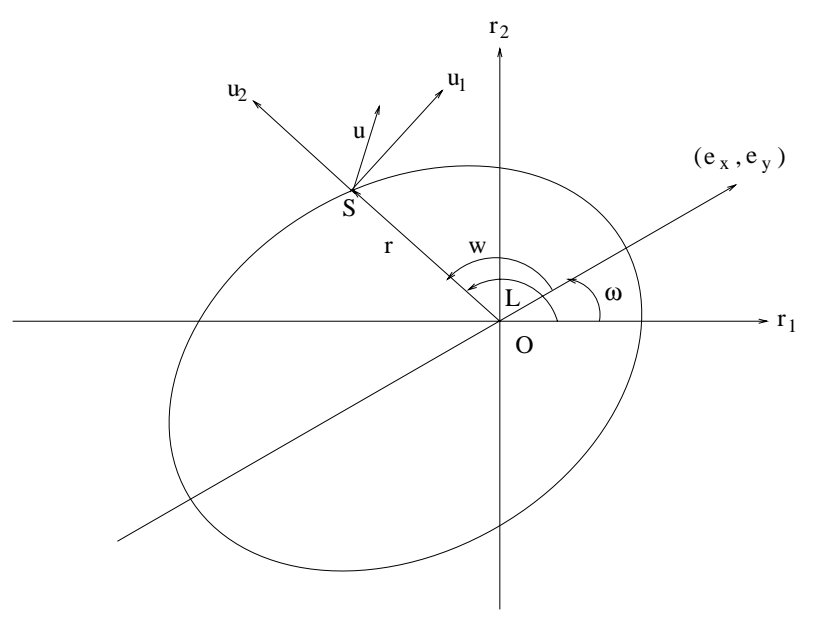

Figure 1.1. Orbital coordinates of a satellite $S$ defining an ellipse of focus $O$ (center of the Earth), semi-latus rectum $P$, eccentricity vector $e=\left(e_{x}, e_{y}\right)$, and true longitude $L=\omega+w$. The axes $\left(r_{1}, r_{2}\right)$ define a fixed geocentric frame whereas $\left(u_{1}, u_{2}\right)$ define the moving frame in which the control is expressed.

when expressing the control in the new moving frame of $\mathbf{R}^{2}$ attached to the satellite and forming an angle $L$ with the canonical one (see Fig. 1.1). The trajectory is assumed to stay in a security zone $A \subset \mathbf{R} \times M^{4}$ of the $t x$-plane defined by

$$
t \geq 0, P \geq \Pi^{0},|e| \leq \varepsilon^{0}
$$

$\left(\Pi^{0}>0,0<\varepsilon^{0}<1\right)$. Similarly, the mass is prescribed to remain greater than the proper mass $\chi^{0}$ of the satellite (mass without fuel)

$$
m \geq \chi^{0}
$$

These path constraints will only be used for controllability and existence, and will be dropped afterwards. There are also boundary constraints (lying in the interior of the sets defined by path constraints at $t=0$ and $t=t_{f}$ )

$$
x(0)=x^{0}, m(0)=m^{0}, h\left(x\left(t_{f}\right)\right)=0
$$

with $h$ a smooth submersion of $M^{4}$ onto $\mathbf{R}^{3}$, as well as a constraint on the maximum modulus of the thrust

$$
|u| \leq T_{\max }
$$

Again, this is equivalent to $u_{1}^{2}+u_{2}^{2} \leq T_{\max }^{2}$. Since we are mainly interested in low-thrust transfers, $T_{\max }$ is assumed to be very small. The performance index to be minimized is the transfer time,

$$
t_{f} \rightarrow \min
$$

The optimal trajectory is sought as an absolutely continuous function in $W_{4}^{1, \infty}\left(\left[0, t_{f}\right]\right)=W^{1, \infty}\left(\left[0, t_{f}\right], \mathbf{R}^{4}\right)$, together with the mass to be found in $W^{1, \infty}\left(\left[0, t_{f}\right]\right)$, whereas the control is sought as an essentially bounded function in $L_{2}^{\infty}\left(\left[0, t_{f}\right]\right)=L^{\infty}\left(\left[0, t_{f}\right], \mathbf{R}^{2}\right)$. For a given maximum thrust $T_{\max }$, the minimum time control problem will be referred to as $(S P)_{T_{\max }}$. It is proved in next section that, no matter how low the thrust may be, the system remains controllable for a small enough proper mass. 


\section{Controllability}

We will use in this section the vector field point of view of [16,17], appealing to the formalism of [11]. Let $f$ be a smooth function defined on the product of an $n$-dimensional manifold $M^{n}$ with a control set $U$ into the tangent bundle $T M$ of $M^{n}$ such that

$$
f(x, u) \in T_{x} M,(x, u) \in M^{n} \times U .
$$

The family of vector fields $\mathcal{F}(U)$ associated with $f$ and $U$ is the subset of $\mathcal{T}(M)$ (Lie algebra of smooth vector fields on $M^{n}$ ) given by $\mathcal{F}(U)=\{f(., u) \mid u \in U\}$. The Lie bracket of two vector fields $X, Y$ is defined by $[X, Y]=X Y-Y X$, and the Poisson bracket of two smooth functions $f, g$ on the cotangent bundle $T^{*} M$ of $M^{n}$ by $\{f, g\}=-\sum_{i=1}^{n} \partial_{x_{i}} f \partial_{p_{i}} g-\partial_{p_{i}} f \partial_{x_{i}} g$ (sign convention in canonical coordinates as in [10]). In the case of control-affine systems with a periodic drift, we have the following controllability result (as a particular case of Th. 5, Chap. 4 in [11]):

Theorem 2.1. Let $\mathcal{F}(U)=\left\{X_{0}+\sum_{i=1}^{m} u_{i} X_{i}, u \in U\right\}$, with $M^{n}$ a connex manifold; then, under the assumptions

(i) $X_{0}$ is periodic;

(ii) the convex hull of $U, \operatorname{co}(U)$, is a neighbourhood of 0 ;

(iii) $\operatorname{Lie}_{x}\left(\left\{X_{0}, X_{1}, \ldots, X_{m}\right\}\right)=T_{x} M, x \in M^{n}$

$\mathcal{F}(U)$ is controllable.

The controllability of the transfer system defined in Section 1 follows, provided the satellite is light enough (for the constant mass model, controllability holds unconditionally):

Proposition 2.2. For any $T_{\max }$, there is a proper mass $\chi^{0}>0$ of the satellite that makes the system controllable.

Proof. We first prove controllability of the constant mass system

$$
\begin{aligned}
\dot{x} & =f_{0}(x)+\gamma_{1} f_{1}(x)+\gamma_{2} f_{2}(x) \\
|\gamma| & \leq T_{\max } / m^{0} .
\end{aligned}
$$

Let $Q^{4}$ be the open submanifold of $M^{4}$ defined by $P>\Pi^{0}$ and $|e|<\varepsilon^{0}$. $f_{0}$ is periodic on $Q^{4}$, since the flow of the drift is the free oscillation of the system which is elliptic $\left(|e|<\varepsilon^{0}<1\right)$. Furthermore, one can check that $\operatorname{Vect}_{x}\left(\left\{f_{1}, f_{2},\left[f_{0}, f_{1}\right],\left[f_{0}, f_{2}\right]\right\}\right)=T_{x} Q$ (see Sect. 3 for explicit computation), which implies $\operatorname{Lie}_{x}\left(\left\{f_{0}, f_{1}, f_{2}\right\}\right)=T_{x} Q$. As the control set is the closed Euclidean ball of radius $T_{\max } / m^{0}$, controllability proceeds from Theorem 2.1. The controllability of the original system with varying mass is then established by setting

$$
\begin{aligned}
& m=m^{0} \exp \left(-\delta \int_{0}^{t}|\gamma| \mathrm{d} s\right)>0 \\
& u=m \gamma
\end{aligned}
$$

where $\gamma$ controls $(2.1,2.2)$. Indeed, $\dot{m}=-\delta|u|$ and $|u| \leq m^{0} T_{\max } / m^{0}=T_{\max }$. If $T$ is the resulting transfer time, the proper mass $\chi^{0}=m^{0} \exp \left(-\delta T T_{\max } / m^{0}\right)>0$ is obviously such that $m \geq \chi^{0}$ on $[0, T]$.

Controllability would be demonstrated exactly in the same way for the 3D model [3]. Furthermore, the result still holds with an additional constraint on the angle of the control (expressed, as before, in the local frame attached to the satellite; see Fig. 2.1).

Corollary 2.3. For any $T_{\max }>0$, for any angle constraint defined by $\alpha>\pi / 2$, the system remains controllable provided the proper mass is small enough. 


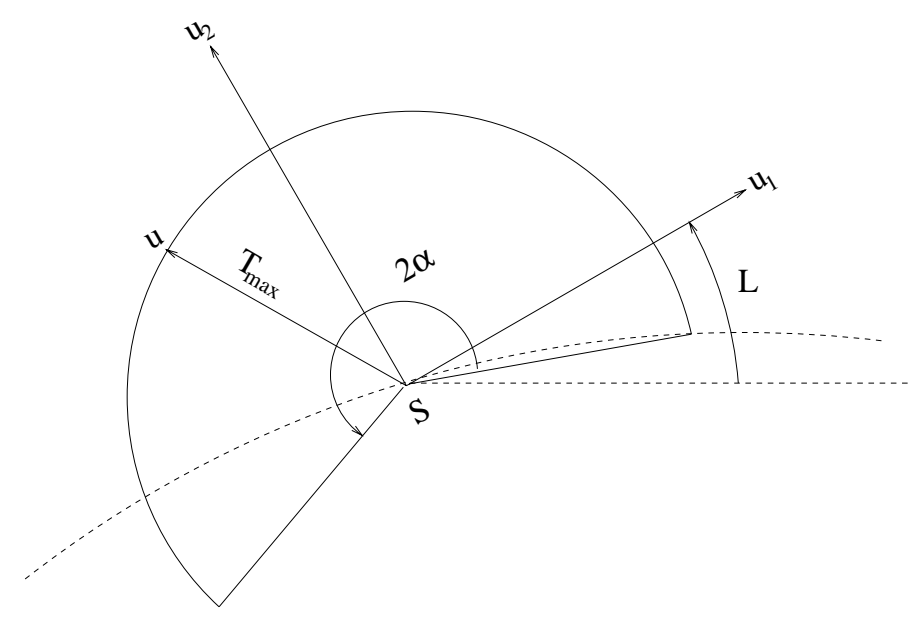

Figure 2.1. The thrust is prescribed to stay in the intersection of the Euclidean ball of radius $T_{\max }$ with a cone attached to the moving frame of half angle $\alpha$ and axis $u_{2}$.

Proof. Indeed, the convex hull of the control set is still a neighbourhood of the origin (here, the satellite) whenever $\alpha$ is strictly greater than $\pi / 2$.

Henceforward, we will assume that $\chi^{0}$ is choosen in order to ensure controllability. Existence for $(S P)_{T_{\max }}$ easily follows from Proposition 2.2:

Corollary 2.4. For any $T_{\max }>0$, there is an optimal control achieving the minimum time transfer.

Proof. The set of admissible trajectories is not empty, because of controllability; hence, we can restrict ourselves to consider transfer times less than a fixed positive $T, T$ big enough. Furthermore, in cartesian coordinates,

$$
(x \mid f(x, m, u))=(r \mid v)-\mu^{0}(v \mid r) /|r|^{3}+(v \mid u) / m \leq|x|^{2}+|x|\left(\mu^{0}\left(1+\varepsilon^{0}\right)^{2} /\left(\Pi^{0}\right)^{2}+T_{\max } / \chi^{0}\right)
$$

so there is a positive constant $C$ such that $(x \mid f(x, m, u)) \leq C\left(1+|x|^{2}\right)$, from where we get that the trajectories remain in a fix compact $K$ of $\mathbf{R} \times M^{4}$. As $U$ is a compact Euclidean ball, any admissible triple $(x, m, u)$ is such that $(t, x, m, u)$ stays into the compact $[0, T] \times K \times\left[\chi^{0}, m^{0}\right] \times U$ almost everywhere (the mass is always decreasing). Finally, since $\widetilde{Q}(x, m)=\widetilde{f}(x, m, U)$ is convex - where $\widetilde{f}(x, m, u)$ is the extended dynamics including the mass variation, $\widetilde{f}(x, m, u)=(f(x, m, u),-\delta|u|)$ - existence is given by Filippov theorem [6].

Properties of optimal controls are studied in next section.

\section{Structure of optimal Controls}

We consider a minimum time control problem with smooth control-affine and non-autonomous dynamics (see $[1,2,16]$ for autonomous cases with scalar controls)

$$
\dot{x}=f_{0}(x)+k(t)\left(u_{1} f_{1}(x)+u_{2} f_{2}(x)\right)
$$

defined on a 4-dimensional manifold $M^{4}$, with endpoint conditions

$$
x(0)=x^{0}, h\left(x\left(t_{f}\right)\right)=0
$$


( $h$ submersion of $M^{4}$ onto $\mathbf{R}^{l}, l \leq 3$ ) and a control constraint (defining a control set $U \subset \mathbf{R}^{2}$ )

$$
|u| \leq T_{\max }\left(T_{\max }>0\right) .
$$

As we shall see at the end of the section, the problem $(S P)_{T_{\max }}$ is a particular case of this one. We make a first assumption on the dynamics (3.1):

(A3.1) $k$ is monotonous and non-vanishing.

The (time and control dependent) Hamiltonian of the problem is

$$
H(t, y, u)=H_{0}(y)+k(t)\left(u_{1} H_{1}(y)+u_{2} H_{2}(y)\right)
$$

where $H_{i}(y)=H_{f_{i}}(y)=p f_{i}(x), i=0,1,2$ and $y=(x, p)$ (canonical coordinates on $T^{*} M$ ); we denote by $Y$ the vector field on $T^{*} M$ associated with $H$ thanks to the canonical symplectic structure of the cotangent bundle [10]. Then, if $\left(t_{f}, x, u\right)$ is solution, Pontryagin maximum principle [11,16] implies that there exists an absolutely continuous integral curve $y=(x, p)$ of $Y$ on $\left[0, t_{f}\right]$

$$
\dot{y}=Y(t, y, u)
$$

and a positive number $p_{0}$ such that

(i) $\left(p_{0}, p\right) \neq 0$, and here, because the criterion is $t_{f}, p \neq 0$ (non-triviality);

(ii) $j_{B}^{*}\left(p_{0} \mathrm{~d} t+H\left(t_{f}\right) \mathrm{d} t-\sum_{i=1}^{4} p_{i}\left(t_{f}\right) \mathrm{d} x_{i}\right)\left(t_{f}, x\left(t_{f}\right)\right)=0$ (transversality);

(iii) $H(t, y, u)=\min _{v \in U} H(t, y, v)$ on $\left[0, t_{f}\right]$ (minimality).

Hereabove, $B^{q}$ is the submanifold of $\mathbf{R} \times M^{4}$ of dimension $q=4-l+1$ defined by the terminal constraint:

$$
B^{q}=\left\{(t, x) \in \mathbf{R} \times M^{4} \mid t>0, h(x)=0\right\}
$$

and $j_{B}^{*}$ is the pull-back of the canonical embedding $j_{B}: B^{q} \rightarrow \mathbf{R} \times M^{4}$. Furthermore, as a function of time, $H$ is absolutely continuous and

$$
\dot{H}=\partial_{t} H(t, y, u)
$$

Now, if we define the switching function $\psi$ along the optimal trajectory by

$$
\psi=\left(H_{1}, H_{2}\right)
$$

because of the minimality of $H$, the optimal control verifies ( $\varepsilon$ denoting the constant sign - by virtue of (A3.1) of $k$ )

$$
u=-\varepsilon T_{\max } \psi /|\psi|
$$

whenever $\psi$ does not vanish, that is outside switching points. Our aim is to study these points. Using the second assumption that

(A3.2) $f_{1}$ and $f_{2}$ commute

we have the first

Proposition 3.1. Under assumptions (A3.1, A3.2), $\psi$ is continuously differentiable.

Proof. $\psi$ is absolutely continuous and $\dot{\psi}=\left(\dot{H}_{1}, \dot{H}_{2}\right)$. Now, we know that

$$
\dot{H}_{i}=\left\{H, H_{i}\right\}=H_{\left[f, f_{i}\right]}=H_{\left[f_{0}, f_{i}\right]}=\left\{H_{0}, H_{i}\right\}
$$


since $\left[f, f_{i}\right]=\left[f_{0}+k(t)\left(u_{1} f_{1}+u_{2} f_{2}\right), f_{i}\right]=\left[f_{0}, f_{i}\right], i=1,2$, in accordance with (A3.2). Consequently, $\dot{\psi}$ is continuous and $\psi$ of class $C^{1}$.

We now require that some of the first Lie brackets of the vector fields defining the dynamics span the whole tangent space when evaluated at one point; more precisely, we assume

(A3.3) $\operatorname{Vect}_{x}\left(\left\{f_{1}, f_{2},\left[f_{0}, f_{1}\right],\left[f_{0}, f_{2}\right]\right\}\right)=T_{x} M, x \in M^{4}$.

Then we have the finiteness of switching points:

Proposition 3.2. Under assumptions (A3.1-A3.3), $\psi$ has finitely many zeros.

Proof. Let $\bar{t}$ be a switching point, $\psi(\bar{t})=0$, and let us suppose that $\dot{\psi}(\bar{t})=0$. Then, at $\bar{t}, H_{i}=\left\{H_{0}, H_{i}\right\}=0$, $i=1,2$, and the covector $p(\bar{t})$ is orthogonal to $f_{1}, f_{2},\left[f_{0}, f_{1}\right]$ and $\left[f_{0}, f_{2}\right]$ at $x(\bar{t})$. By virtue of $($ A3.3), $p(\bar{t})=0$, so that $p \equiv 0$ which contradicts the maximum principle. Thus, $\dot{\psi}(\bar{t}) \neq 0$ and every switching point is isolated on the compact $\left[0, t_{f}\right]: \psi$ has only a finite number of zeros.

Concerning the geometry of switchings, we have:

Proposition 3.3. Under assumptions (A3.1-A3.3), any switching is of angle $\pi$.

Proof. Let $\bar{t} \in\left[0, t_{f}\right]$ be such that $\psi(\bar{t})=0$. We know by Proposition 3.2 that $\dot{\psi}(\bar{t}) \neq 0$; since $\psi$ is continuously differentiable, its graph has a tangent at the origin and the conclusion follows from Figure 3.1.

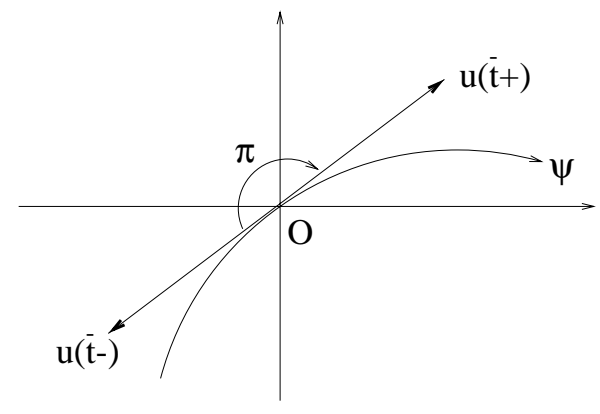

FIGURE 3.1. Switching of angle $\pi$ of the optimal control.

Corollary 3.4. Under assumptions (A3.1-A3.3), $x, u$ and $p$ are smooth on $\left[t_{i}, t_{i+1}\right]$, for any consecutive switchings $t_{i}<t_{i+1}$.

Proof. Let $t_{i}<t_{i+1}$ be two consecutive switchings; thanks to the regularity of the data, $y=(x, p)$ and $u-$ which is smoothly defined as a function of $y$ outside switchings by $u=-\varepsilon T_{\max }\left(H_{1}, H_{2}\right) /\left|\left(H_{1}, H_{2}\right)\right|-$ are smooth on ]$t_{i}, t_{i+1}\left[\right.$. Now, by virtue of Proposition 3.3, $u$ admits left and right limits at $t_{i}, t_{i+1}$; therefore, as $\dot{x}=f(t, x, u)$ and $\dot{p}=-p \partial_{x} f(t, x, u), \dot{x}$ and $\dot{p}$ also do: by finite increments, $x$ and $p$ are of a class $C^{1}$ on the closed interval $\left[t_{i}, t_{i+1}\right]$. In as much as the derivatives of order $(k+1)$ of $x$ and $p$ only depend on derivatives of $u$ up to order $k$, an immediate recurrence concludes the proof.

Finally, we give bounds on the number of consecutive switchings located at special points (that will have a natural geometric interpretation on the transfer problem, see Cor. 3.7). Namely, if $\mathcal{C}$ denotes the finite set of zeros of $\psi$, let

$$
\mathcal{C}_{2}=\left\{t \in \mathcal{C} \mid f_{0}(x(t)) \in \operatorname{Vect}_{x(t)}\left(\left\{f_{1}, f_{2},\left[f_{0}, f_{2}\right]\right\}\right)\right\}
$$


Then, under the last two assumptions

(A3.4) $\operatorname{Vect}_{x}\left(\left\{f_{0}, f_{1}, f_{2},\left[f_{0}, f_{1}\right]\right\}\right)=T_{x} M, x \in M^{4}$;

(A3.5) $p_{0}>0$ (constraint qualification)

the following is true:

Proposition 3.5. Under assumptions (A3.1-A3.5), if $k \dot{k} \leq 0$, there cannot be consecutive switchings in $\mathcal{C}_{2}$. If $k \dot{k}>0$, there can be at most three consecutive switchings in $\mathcal{C}_{2}$.

Proof. According to (A3.3), there are smooth functions $\alpha_{1}, \ldots, \alpha_{4}$ in $\mathcal{D}(M)$ such that

$$
f_{0}=\alpha_{1} f_{1}+\alpha_{2} f_{2}+\alpha_{3}\left[f_{0}, f_{1}\right]+\alpha_{4}\left[f_{0}, f_{2}\right]
$$

If $\bar{t}$ is a switching point, $H(\bar{t})=H_{0}(\bar{t})=\left(\alpha_{3}\left\{H_{0}, H_{1}\right\}+\alpha_{4}\left\{H_{0}, H_{2}\right\}\right)(\bar{t})$. Moreover, if $\bar{t} \in \mathcal{C}_{2}, H(\bar{t})=$ $\alpha_{4}(\bar{t})\left\{H_{0}, H_{2}\right\}(\bar{t})=\alpha_{4}(\bar{t}) \dot{\psi}_{2}(\bar{t})$. Now, by (3.2), $\dot{H}=\dot{k}(t)\left(u_{1} H_{1}+u_{2} H_{2}\right)$, whose sign is constant (opposite to the sign of $k \dot{k}$ ). If $k \dot{k} \leq 0, H$ is increasing towards $H\left(t_{f}\right)=-p_{0}$ (transversality condition of the maximum principle) and therefore is strictly negative on $\left[0, t_{f}\right]$ by (A3.5). Then, if $\bar{t}_{1}<\bar{t}_{2}$ are two consecutive switching instants in $\mathcal{C}_{2}$, we have

$$
\alpha_{4}\left(\bar{t}_{1}\right) \alpha_{4}\left(\bar{t}_{2}\right) \dot{\psi}_{2}\left(\bar{t}_{1}\right) \dot{\psi}_{2}\left(\bar{t}_{2}\right)=H\left(\bar{t}_{1}\right) H\left(\bar{t}_{2}\right)>0
$$

As (A3.4) implies that $\alpha_{4}$ never vanishes along the optimal trajectory, we conclude that $\dot{\psi}_{2}\left(\bar{t}_{1}\right) \dot{\psi}_{2}\left(\bar{t}_{2}\right)>0$, which is impossible $\left(\bar{t}_{1}\right.$ and $\bar{t}_{2}$ being two consecutive zeros of $\psi_{2}$ ). If $k \dot{k}>0$ (which is more restrictive than the negation of the case $k \dot{k} \leq 0), H$ is strictly decreasing towards $H\left(t_{f}\right)=-p_{0}<0$ ( $H$ cannot be constant on any subinterval of $\left[0, t_{f}\right]$ since, by Prop. 3.2, there is a finite number of switchings) and vanishes at most once. Suppose this is the case, and let $t^{*} \in\left[0, t_{f}\right]$ be the zero (otherwise the sign of $H$ is constant and the previous argument holds), then the former analysis implies there cannot be consecutive switchings in $\mathcal{C}_{2}$ either in $\left[0, t^{*}[\right.$ or in $\left.] t^{*}, t_{f}\right]$. As $t^{*}$ may belong to $\mathcal{C}_{2}$, there can be up to three consecutive switchings in $\mathcal{C}_{2}$, no more. The proof is complete.

In order to apply the previous results to the transfer problem, we need to suppose

(I1) any optimal couple $(x, m)$ stays into the interior of the path constraints $(1.7,1.8)$

Then, it is enough to eliminate the mass variation by proving:

Proposition 3.6. Under assumption (I1), any optimal control $u$ of $(S P)_{T_{\max }}$ is such that $|u|=T_{\max }$ almost everywhere.

Indeed, this proposition implies that $m(t)=m^{0}-\delta T_{\max } t$, and the transfer problem falls under the realm of the previous one by posing $k=1 / m$.

Proof of Proposition 3.6. The Hamiltonian of the initial problem, including the mass variation, is

$$
\widetilde{H}=H_{0}+1 / m\left(u_{1} H_{1}+u_{2} H_{2}\right)-\delta p_{m}|u|
$$

with $p_{m}$ adjoint state associated with $m$. Hence, $\dot{p}_{m}=-\partial_{m} \widetilde{H}$,

$$
\dot{p}_{m}=1 / m^{2}\left(u_{1} H_{1}+u_{2} H_{2}\right)
$$

which is negative since the control minimizes $\widetilde{H}$ on the closed Euclidean ball of radius $T_{\max }$ : $p_{m}$ decreases towards $p_{m}\left(t_{f}\right)$ which is zero by transversality. As a result, $p_{m}$ is positive. Then, with the same notations as before, $u=-T_{\max } \psi /|\psi|$ whenever $\psi$ does not vanish. Now, $\psi$ is still continuously differentiable, 
and $\dot{\psi}=\left(\left\{H_{0}, H_{1}\right\},\left\{H_{0}, H_{2}\right\}\right)$. Indeed, taking $\widetilde{x}=(x, m), \widetilde{p}=\left(p, p_{m}\right),(\widetilde{x}, \widetilde{p})$ local coordinates of $\widetilde{y} \in$ $T^{*}\left(M \times \mathbf{R}_{+} \backslash\{0\}\right), \widetilde{H}_{i}=H_{\widetilde{f}_{i}}$ with $\widetilde{f}_{i}(\widetilde{x})=\left(f_{i}(x), 0\right), i=0,1,2$, one verifies

$$
\widetilde{H}_{i}(\widetilde{y})=H_{i}(y),\left[\widetilde{f}_{0}, \widetilde{f}_{i}\right](\widetilde{x})=\left(\left[f_{0}, f_{i}\right](x), 0\right)
$$

so that, along the optimal trajectory,

$$
\dot{H}_{i}=\dot{\widetilde{H}}_{i}=\left\{\widetilde{H}_{0}, \widetilde{H}_{i}\right\}=\left\{H_{0}, H_{i}\right\} .
$$

Then, if $\psi(\bar{t})=0$ and $\dot{\psi}(\bar{t})=0$, by the same argument as in Proposition 3.2,p( $\bar{t})=0$. Moreover, as $\dot{p}=$ $-p\left(f_{0}^{\prime}+1 / m\left(u_{1} f_{1}^{\prime}+u_{2} f_{2}^{\prime}\right)\right), p \equiv 0$ by linearity. Then, $p_{m}$ is also identically zero because of (3.4) (plus the fact that $p_{m}\left(t_{f}\right)=0$ ), that is $\widetilde{p} \equiv 0$ which is impossible (since the criterion is $t_{f}$ ). As before, we conclude that $\psi$ has only finitely many zeros, and that $|u|=T_{\max }$ holds almost everywhere.

With the assumption

(I1') there is constraint qualification on $(S P)_{T_{\max }}$

Proposition 3.5 has the following corollary.

Corollary 3.7. Under assumptions (I1-I1'), in the constant mass case $(\delta=0)$ there cannot be consecutive switchings at the perigee or at the apogee. In the variable mass case $(\delta>0)$, there are at most three consecutive switchings at the perigee or at the apogee. In any case, switchings are of angle $\pi$.

Proof. One can check on (1.4-1.6) that $f_{1}$ and $f_{2}$ commute, and that, in orbital coordinates:

$$
\begin{aligned}
{\left[f_{0}, f_{1}\right]=} & W^{2} / P\left(\cos L \partial_{e_{x}}+\sin L \partial_{e_{y}}\right) \\
{\left[f_{0}, f_{2}\right]=} & 2\left(e_{x} \sin L-e_{y} \cos L\right) \partial_{P}+W^{2} / P\left(-\sin L-\sin L / W+\left(e_{x}+\cos L\right)\left(e_{x} \sin L-e_{y} \cos L\right) / W^{2}\right) \partial_{e_{x}} \\
& +W^{2} / P\left(\cos L+\cos L / W+\left(e_{y}+\sin L\right)\left(e_{x} \sin L-e_{y} \cos L\right) / W^{2}\right) \partial_{e_{y}}-W / P \partial_{L} .
\end{aligned}
$$

Since $P$ and $W$ are strictly positive on $M^{4}$ the assumptions of Propositions 3.3 and 3.5 hold. In particular, any switching is of angle $\pi$. Moreover, at the perigee or at the apogee, the eccentricity vector $e=\left(e_{x}, e_{y}\right)$ is colinear with the position vector so that the determinant $e_{x} \sin L-e_{y} \cos L$ is zero. Then, by (1.4-1.6) and (3.6), $f_{0}$ belongs to the subspace generated by $f_{1}$ and $\left[f_{0}, f_{2}\right]$ : with the previous notations, any switching point located at the perigee or at the apogee is in $\mathcal{C}_{2}$. Hence, by Theorem 2.1, if $\delta=0$ (constant mass) then

$$
k(t)=1 / m^{0}, t \in\left[0, t_{f}\right]
$$

and we cannot have consecutive switchings at the perigee or at the apogee $(k \dot{k}=0)$. If $\delta>0$ (variable mass) then

$$
k(t)=1 /\left(m^{0}-\delta T_{\max } t\right), t \in\left[0, t_{f}\right]
$$

and we have at most three consecutive switchings at the perigee or at the apogee $(k \dot{k}>0)$.

As we shall see in Section 5 , this result is of practical importance since the numerical experiments demonstrate that the possible switchings occur at the perigee. A similar analysis can be done on the 3D model [3]. We depict in the following section the main features of the method used for the numerical computation.

\section{Parametric technique}

We consider the abstract optimization problem $(O)$ of minimizing a performance index $J$, defined on a Banach space $\mathcal{Z}$

$$
J(z) \rightarrow \min
$$


under the constraints

$$
\begin{aligned}
& z \in \mathcal{Z}_{a d} \subset \mathcal{Z} \\
& F(z)=0
\end{aligned}
$$

where $F$ is a mapping from $\mathcal{Z}$ to another Banach space $\mathcal{Y}$ imbedded into a Hilbert space $\widehat{\mathcal{Y}}$. To $(O)$ we associate the parametric optimization problem $(O)_{\beta}(\beta$ is the parameter) consisting in minimizing

$$
1 / 2\|\alpha\|_{\hat{\mathcal{Y}}}^{2} \rightarrow \min
$$

under the parametric constraints

$$
\begin{aligned}
(z, \alpha) & \in \mathcal{Z}_{a d} \times \mathcal{Y} \\
F(z) & =S \alpha \\
J(z) & =\beta
\end{aligned}
$$

with $S$ a fixed linear operator on $\mathcal{Y}$. We then define the function $\phi$ as the value function of the family $(O)_{\beta}$, that maps $\beta$ to the optimal value $\phi(\beta) \in \overline{\mathbf{R}}$ of $(O)_{\beta}$. Thus, $\phi(\beta)$ gives a measurement of the non-controllability of the initial problem, when the criterion is enforced to take the value $\beta$ by (4.3), with respect to the equality constraints selected by the operator $S$ in (4.2). Then, the optimal value of $(O)$ is the smallest $\beta$ for which controllability occurs: actually, if we define the problem $(E)$ of finding the (unique) first root of $\phi$, we have:

Proposition 4.1. The problems $(O)$ and $(E)$ are equivalent in that any solution to one of them induces a solution to the other.

Proof. If $\bar{z}$ is solution to $(O), \bar{\beta}=J(\bar{z})$ is solution to $(E)$; indeed, if there is a $\widetilde{\beta}$ strictly smaller than $\bar{\beta}$ and such that $\phi(\widetilde{\beta})=0$, there is also a pair $(\widetilde{z}, \widetilde{\alpha})$ solution to $(O)_{\tilde{\beta}}$. As $\phi(\widetilde{\beta})=0, \widetilde{\alpha}=0$ and $\widetilde{z}$ is admissible for $(O)$, which contradicts the optimality of $\bar{z}$ since $J(\widetilde{z})=\widetilde{\beta}<\bar{\beta}=J(\bar{\beta})$. Conversely, if $\bar{\beta}$ is solution to $(E), \phi(\bar{\beta})=0$ so it exists $\bar{z} \in \mathcal{Z}$ such that $(\bar{z}, 0)$ is solution to $(O)_{\bar{\beta}}$; then, $\bar{z}$ is admissible for $(O)$, and furthermore optimal. Otherwise, one could find an admissible $\widetilde{z}$ verifying $J(\widetilde{z})<J(\bar{z})$ and $\widetilde{\beta}=J(\widetilde{z}$ ) would be a root of $\phi$ (admissibility of $\widetilde{z}$ ) strictly smaller than $\bar{\beta}$.

Let then $(O C P)$ be a minimum time problem

$$
t_{f} \rightarrow \min
$$

with dynamics on a smooth $n$-dimensional open submanifold $M^{n}$ of $\mathbf{R}^{n}$

$$
\dot{x}=f(t, x, u)
$$

with end-point, path and control constraints

$$
\begin{aligned}
& x(0)=x^{0}, h\left(x\left(t_{f}\right)\right)=0 \\
& (t, x) \in A, u \in U(t, x)
\end{aligned}
$$

where $f$ and $h$ are smooth functions ( $h$ submersion of $M^{n}$ onto $\mathbf{R}^{l}, l \leq n-1$ ). We also require that the subsets $A \subset \mathbf{R} \times M^{n}$ and $N=\left\{(t, x, u) \in \mathbf{R} \times M^{n} \times U \mid(t, x) \in A, u \in \bar{U}(t, x)\right\}$ be closed. $(O C P)$ is obviously a 
particular case of $(O)$ since, using an homothety to recast the problem on $[0,1]$, we can set $z=\left(t_{f}, x, u\right) \in \mathcal{Z}$ with

$$
\begin{aligned}
& \mathcal{Z}=\mathbf{R} \times \mathcal{X} \times \mathcal{U}, \mathcal{X}=W_{n}^{1, \infty}([0,1]), \mathcal{U}=L_{m}^{\infty}([0,1]) \\
& \mathcal{Y}=L_{n}^{\infty}([0,1]) \times \mathbf{R}^{n} \times \mathbf{R}^{l}, \widehat{\mathcal{Y}}=L_{n}^{2}([0,1]) \times \mathbf{R}^{n} \times \mathbf{R}^{l} \\
& \mathcal{Z}_{a d}=\left\{z \in \mathcal{Z} \mid t_{f} \geq 0,\left(t_{f} t, x\right) \in A, u \in U\left(t_{f} t, x\right)\right\} \\
& J(z)=t_{f}, F(z)=\left[\begin{array}{l}
\dot{x}-t_{f} f\left(t_{f} t, x, u\right) \\
x(0)-x^{0} \\
h(x(1))
\end{array}\right] .
\end{aligned}
$$

Several choices are then possible for the linear operator $S$ in (4.2), depending on the constraint one wants to lay the emphasis on. For instance, in [15], the constraint on the dynamics was selected and $\phi(\beta)$ was the value function of the parametric problem with fixed final time $\left(\beta=t_{f}\right.$ since the criterion is the free final time)

$$
\begin{aligned}
& 1 / 2\|\alpha\|_{L_{n}^{2}}^{2} \rightarrow \min \\
& \dot{x}=f(t, x, u)+\alpha, t \in[0, \beta] \\
& x(0)=x^{0}, h(x(\beta))=0 \\
& (t, x) \in A, u \in U(t, x)
\end{aligned}
$$

where $\alpha \in L_{n}^{\infty}([0, \beta])$, which was added to the right-hand side of the dynamics, acted as a fictitious control. Here, we make a different choice, emphasizing the role of the terminal constraint $h\left(x\left(t_{f}\right)\right)=0$. As a result, $\phi(\beta)$ becomes the value function of the problem $(O C P)_{\beta}$ with fixed final time defined on $[0, \beta]$ (where the slackness variable $\alpha$ disappears)

$$
\begin{aligned}
& 1 / 2|h(x(\beta))|^{2} \rightarrow \min \\
& \dot{x}=f(t, x, u), t \in[0, \beta] \\
& x(0)=x^{0} \\
& (t, x) \in A, u \in U(t, x) .
\end{aligned}
$$

The problem obtained is much simpler than the original one since it does not include a constraint on the terminal state anymore. This peculiarity will allow an easy derivation of properties of the value function $\phi$. The following basic assumptions on $(O C P)$ will be usefull, mainly for the existence of measurable selectors and optimal controls:

(A4.1) the set of admissible triples $\left(t_{f}, x, u\right)$ for $(O C P)$ is non-empty;

(A4.2) the $t x u$-space $N$ is compact;

(A4.3) $Q(t, x)=f(t, x, U(t, x))$ is convex for any $(t, x)$ in $\mathbf{R} \times M^{n}$.

Under the previous assumptions, we know by measurable selection [6] that, if $x \in W_{n}^{1, \infty}\left(\left[t_{1}, t_{2}\right]\right)$ verifies $\dot{x} \in$ $Q(t, x),(t, x) \in A$, there is a measurable control $u \in L_{m}^{\infty}\left(\left[t_{1}, t_{2}\right]\right)$ such that

$$
\dot{x}=f(t, x, u), u \in U(t, x) .
$$

We then make the following structural assumption on $(O C P)$ :

(A4.4) for any $\left(t_{1}, x_{1}\right) \in A$ and $t_{2}>t_{1}$, there is a trajectory $x \in W_{n}^{1, \infty}\left(\left[t_{1}, t_{2}\right]\right)$ such that

$$
\begin{aligned}
& \dot{x}=Q(t, x),(t, x) \in A \\
& x\left(t_{1}\right)=x_{1}, h\left(x\left(t_{1}\right)\right)=h\left(x\left(t_{2}\right)\right) .
\end{aligned}
$$

This assumption means that, from a given point, it is possible to steer the system to any later time by means of an admissible trajectory without changing the value of the terminal constraint. This property, crucial for the analysis hereafter, will be straightforwardly fulfilled in the transfer case. We briefly state some basic facts [5] about $\phi$ needed to study the convergence of the method. 
Proposition 4.2. Under assumptions (A4.1-A4.4), $\phi$ is finite, decreasing, and zero after its first root $\bar{\beta}$.

Proof. Obvious.

Proposition 4.3. Under assumptions (A4.1-A4.4), $\phi$ is Lipschitz continuous. Moreover, if there is an optimal trajectory two times differentiable at $\bar{t}_{f}, \phi^{\prime}(\bar{\beta})=0$ and $\phi(\beta)=O\left((\beta-\bar{\beta})^{2}\right)$ in the neighbourhood of $\bar{\beta}$.

Proof. Let $\beta_{1}$ and $\beta_{2}$ be such that $0 \leq \beta_{1} \leq \beta_{2} \leq \bar{\beta}$. For any $\beta \in[0, \bar{\beta}]$, denote $(x(., \beta), u(., \beta))$ a solution of $(O C P)_{\beta}$. Since $\dot{x}(t, \beta)=f(t, x(t, \beta), u(t, \beta))$ on $[0, \beta]$ (a.e.), since $f$ is continous and $N$ is compact, the family $(x(., \beta))_{\beta}$ is equilipschitzian on $[0, \bar{\beta}](x(., \beta)$ is extended to $[0, \bar{\beta}]$ by constancy and continuity). As the trajectories remain in a fix compact $\left(N\right.$ is compact, thus $A$ also), the family $\left(\frac{1}{2}|h(x(., \beta))|^{2}\right)_{\beta}$ is also equilipschitzian and we can find a positive constant $k$ independent of $\beta$ such that:

$$
\left.\left|\frac{1}{2}\right| h\left(x\left(t_{1}, \beta\right)\right)\right|^{2}-\frac{1}{2}\left|h\left(x\left(t_{2}, \beta\right)\right)\right|^{2}|\leq k| t_{1}-t_{2} \mid .
$$

Now, since $\phi$ is decreasing by the previous proposition, we have

$$
0 \leq \phi\left(\beta_{1}\right)-\phi\left(\beta_{2}\right) \leq \frac{1}{2}\left|h\left(x\left(\beta_{1}, \beta_{2}\right)\right)\right|^{2}-\frac{1}{2}\left|h\left(x\left(\beta_{2}, \beta_{2}\right)\right)\right|^{2}
$$

for the restriction of $\left(x\left(., \beta_{2}\right), u\left(., \beta_{2}\right)\right)$ to $\left[0, \beta_{1}\right]$ is admissible for $(O C P)_{\beta_{1}}$. We get from (4.4) and (4.5) that

$$
\left|\phi\left(\beta_{1}\right)-\phi\left(\beta_{2}\right)\right| \leq k\left|\beta_{1}-\beta_{2}\right|
$$

and $\phi$ is Lipschitz. If $\bar{x}$ is an optimal trajectory two times differentiable at $\bar{t}_{f}=\bar{\beta}$, if $\psi(\beta)=1 / 2|h(\bar{x}(\beta))|^{2}$,

$$
0 \leq \frac{\phi(\beta)-\phi(\bar{\beta})}{(\beta-\bar{\beta})^{2}} \leq \frac{1 / 2|h(\bar{x}(\beta))|^{2}}{(\beta-\bar{\beta})^{2}} \rightarrow 1 / 2 \psi^{\prime \prime}(\bar{\beta})
$$

when $\beta \rightarrow \bar{\beta}$ since $\psi(\bar{\beta})=\psi^{\prime}(\bar{\beta})=0\left(c f . h\left(\bar{x}\left(\bar{t}_{f}\right)\right)=0\right)$, which concludes the proof.

The search for the first zero of $\phi$ by an iterative process generates a sequence $\left(\beta_{k}\right)_{k}$ converging towards $\bar{\beta}$. If $\left(x_{k}, u_{k}\right)$ denotes a solution to $(O C P)_{\beta_{k}}$, one may then wish that the last sequence would tend-in some sense-to a couple $(\bar{x}, \bar{u})$ such that $(\bar{\beta}, \bar{x}, \bar{u})$ be solution to $(O C P)$. In this regard, we have the first

Proposition 4.4. Under assumptions (A4.1-A4.4), if $\left(\beta_{k}\right)_{k}$ tends to $\bar{\beta}$, up to a subsequence $\left(x_{k}\right)_{k}$ converges uniformly towards an optimal trajectory $\bar{x}$ of $(O C P)$ in $C_{n}^{0}([0, \bar{\beta}])$.

Proof. The proof goes along the lines of the classical Filippov theorem. The assumptions ensure that $\left(x_{k}\right)_{k}$ is equilipschitzian, and so equicontinuous (as before, each $x_{k}$ is extended by constancy and continuity on $[0, \bar{\beta}]$ ). Since the $t x u$-space $N$ is compact, $\left(x_{k}\right)_{k}$ is also equibounded: by Ascoli theorem, up to a subsequence, $\left(x_{k}\right)_{k}$ has a uniform limit $\bar{x}$ in $C_{n}^{0}([0, \bar{\beta}])$. The closure of an equilipschitzian subset being equilipschitzian, $\bar{x}$ is also Lipschitz. Besides, since $\dot{x}_{k} \in Q\left(t, x_{k}\right)$, since $N$ is compact and since $Q(t, x)$ is convex, $\dot{\bar{x}}$ belongs to $Q(t, \bar{x})$ almost everywhere on $[0, \bar{\beta}]$ (closure Th. 8.6.i of [6]). Of course, $(t, \bar{x}) \in A$ because $A$ is closed and $\bar{x}(0)=x^{0}$ since $x_{k}(0)=x^{0}$. Finally, $x_{k}\left(\beta_{k}\right) \rightarrow \bar{x}(\bar{\beta})$ thanks to the equicontinuity of $\left(x_{k}\right)_{k}$, from where we get that $h(\bar{x}(\bar{\beta}))=0$ since $1 / 2\left|h\left(x_{k}\left(\beta_{k}\right)\right)\right|^{2}=\phi\left(\beta_{k}\right) \rightarrow 0$ (continuity of $\phi$, Prop. 4.3). The trajectory $\bar{x}$ is then admissible for $(O C P)$, and hence optimal by measurable selection.

To obtain a convergence result in the control, we assume that the dynamics can be smoothly inverted in the following way (notion of système plat in [8]):

(A4.5) there are smooth functions $R$ and $S, R(t, x) \in \mathcal{L}\left(\mathbf{R}^{n}, \mathbf{R}^{m}\right)$ and $S(t, x) \in \mathbf{R}^{m}$, such that whenever $y=f(t, x, u)$, one has $u=R(t, x) y+S(t, x)$. 
This assumption holds for instance as soon as the dynamics is control-affine, $\dot{x}=f_{0}(t, x)+B(t, x) u$, with $B(t, x)$ an embedding. Then, we have:

Proposition 4.5. Under assumptions (A4.1-A4.5), if $\left(\beta_{k}\right)_{k}$ tends to $\bar{\beta}$, up to a subsequence $\left(x_{k}, u_{k}\right)_{k}$ converges towards an optimal couple $(\bar{x}, \bar{u})$ for $(O C P)$, uniformly in $C_{n}^{0}([0, \bar{\beta}])$ for the state, weakly-* in $L_{m}^{\infty}([0, \bar{\beta}])$ for the control (the controls $u_{k}$ being extended by zero outside $\left[0, \beta_{k}\right]$ ).

Proof. According to Proposition 4.4, up to a subsequence, $\left(x_{k}\right)_{k}$ converges uniformly to an optimal state $\bar{x}$ of $(O C P)$. By measurable selection, there is an essentially bounded $\bar{u}$ such that $(\bar{\beta}, \bar{x}, \bar{u})$ be solution to $(O C P)$, and (A4.5) implies that

$$
\bar{u}=R(t, \bar{x}) \dot{\bar{x}}+S(t, \bar{x})
$$

As $C_{n}^{0}([0, \bar{\beta}])$ is continuously embedded in the space of distributions, $x_{k} \rightarrow \bar{x}$ in $\mathcal{D}_{n}^{\prime}(] 0, \bar{\beta}[)$ so $\dot{x}_{k} \rightarrow \dot{\bar{x}} \in L_{n}^{\infty}([0, \bar{\beta}])$ in the distributions sense. Since $\dot{x}_{k} \in Q\left(t, x_{k}\right),\left(\dot{x}_{k}\right)_{k}$ is bounded in $L_{n}^{\infty}([0, \bar{\beta}])$ and $\dot{x}_{k} \rightarrow \dot{\bar{x}}$ weakly-* with $\left(\dot{x}_{k}\right)_{k}$ equicontinuous. Let then $\varphi$ belong to $L_{m}^{1}([0, \bar{\beta}])$; as $R$ and $S$ are smooth, if $\chi_{k}$ is the indicator function of [0, $\left.\beta_{k}\right]$, ${ }^{t} R\left(t, x_{k}\right) \varphi \chi_{k} \rightarrow{ }^{t} R(t, \bar{x}) \varphi$ and $\left(S\left(t, x_{k}\right) \chi_{k} \mid \varphi\right) \rightarrow(S(t, \bar{x}) \mid \varphi)$, respectively in $L_{n}^{1}([0, \bar{\beta}])$ and $L^{1}([0, \bar{\beta}])$ by Lebesgue theorem. Hence, $S\left(t, x_{k}\right) \chi_{k} \rightarrow S(t, \bar{x})$ weakly-* and, $\left(\dot{x}_{k}\right)$ being weakly-* convergent and equicontinuous,

$$
\left\langle\dot{x}_{k},{ }^{t} R\left(t, x_{k}\right) \varphi \chi_{k}\right\rangle_{L^{\infty}, L^{1}} \rightarrow\left\langle\dot{\bar{x}},{ }^{t} R(t, \bar{x}) \varphi\right\rangle_{L^{\infty}, L^{1}}
$$

that is $\left\langle R\left(t, x_{k}\right) \dot{x}_{k} \chi_{k}, \varphi\right\rangle_{L^{\infty}, L^{1}} \rightarrow\langle R(t, \bar{x}) \dot{\bar{x}}, \varphi\rangle_{L^{\infty}, L^{1}}$. Since, according to (A4.5), the extension of $u_{k}$ to $[0, \bar{\beta}]$ is equal to $\left(R\left(t, x_{k}\right) \dot{x}_{k}+S\left(t, x_{k}\right)\right) \chi_{k}$, we conclude that $u_{k} \rightarrow \bar{u}$ weakly-* in $L_{m}^{\infty}([0, \bar{\beta}])$.

Finally, if

(A4.6) $U(t, x)=U(t)$ and, for $\beta<\bar{\beta}$, any optimal trajectory $x(., \beta)$ of $(O C P)_{\beta}$ is interior to the path constraint $(t, x) \in A$

Pontryagin maximum principle holds (see Sect. 3$)$, and to any optimal couple $(x(., \beta), u(., \beta))$ of $(O C P)_{\beta}$ we can associate a unique absolutely continuous adjoint state $p(., \beta)$ and a positive number $p_{0}$ such that, in particular, the transversality condition hereafter is true:

$$
p(\beta, \beta)=p_{0}{ }^{t} h(x(\beta, \beta)) h^{\prime}(x(\beta, \beta))
$$

For $\beta<\bar{\beta}$, there is constraint qualification $\left(p_{0}>0\right)$ and we can take $p_{0}=1$ in $(4.6)\left(p_{0}=0\right.$ would imply $\left.p \equiv 0\right)$. Then we also get a convergence result on the adjoint state:

Proposition 4.6. Under assumptions (A4.1-A4.6), if $\left(\beta_{k}\right)_{k}$ tends to $\bar{\beta},\left(p_{k}\right)_{k}$ converges uniformly towards 0 in $C_{n}^{0}([0, \bar{\beta}])$ (with $p_{k}$ extended by constancy and continuity on $\left.[0, \bar{\beta}]\right)$.

Proof. The adjoint state $p_{k}$ verifies the linear equation $\dot{p}_{k}=-p_{k} \partial_{x} f\left(t, x_{k}, u_{k}\right)$ on $\left[0, \beta_{k}\right]$; since $f$ is smooth on the compact $t x u$-space $N$, there is a constant $K$ such that $\left|\partial_{x} f\left(t, x_{k}, u_{k}\right)\right| \leq K$, and

$$
\left\|p_{k}\right\|_{\infty} \leq \exp (K \bar{\beta})\left|p_{k}\left(\beta_{k}\right)\right|
$$

Now, $p_{k}\left(\beta_{k}\right)={ }^{t} h\left(x_{k}\left(\beta_{k}\right)\right) h^{\prime}\left(x_{k}\left(\beta_{k}\right)\right)$ by $(4.6)$, and $h\left(x_{k}\left(\beta_{k}\right)\right) \rightarrow 0$ since $\phi\left(\beta_{k}\right) \rightarrow 0:\left(h^{\prime}\left(x_{k}\left(\beta_{k}\right)\right)\right)_{k}$ being bounded, $p_{k}\left(\beta_{k}\right) \rightarrow 0$ which yields the desired conclusion according to $(4.7)$.

In order to apply these results to the transfer problem, we only need to check assumptions (A4.4, A4.5). Indeed, we saw in Section 2 that the system is controllable (Prop. 2.2) and that the admissible trajectories remain in a fix compact subset (Cor. 2.4). Moreover, we dropped the path constraints (Assump. (I1)) so (A4.6) 
is valid. Now, concerning (A4.4), the verification is obvious: in orbital coordinates, the terminal constraints defined by (1.9) write

$$
P\left(t_{f}\right)=P^{f}, e_{x}\left(t_{f}\right)=e_{x}^{f}, e_{y}\left(t_{f}\right)=e_{y}^{f}
$$

in such a way that the final longitude $L^{f}$ is free; as a consequence, from any point, one can always reach a later time without altering the value of the terminal constraint by means of a zero control, since then the system oscillates freely (elliptic motion) and only the unconstrained longitude is changed. As for assumption (A4.5), if $y=f_{0}(x)+1 / m\left(u_{1} f_{1}(x)+u_{2} f_{2}(x)\right)$, clearly enough $u=m\left({ }^{t} B(x) B(x)\right)^{-1}{ }^{t} B(x)\left(y-f_{0}(x)\right)$ with $B=\left[f_{1} f_{2}\right]$. As a conclusion, all the previous results hold under the sole assumption (I1) on $(S P)_{T_{\max }}$. In particular, Proposition 4.3 implies that $\phi$ has a quadratic behaviour in the neighbourhood of the solution since, by virtue of Corollary 3.4, any optimal trajectory is smooth near $\bar{t}_{f}$. The corresponding numerical results are presented in the last section.

\section{Numerical RESUlts}

In accordance with Section 3 results (Prop. 3.6), we use the non-autonomous formulation for $(S P)_{T_{\max }}$ where the mass is explicitly given by $m(t)=m^{0}-\delta T_{\max } t$. Then, $\phi$ is the value function of the parametric problem $(S P)_{T_{\max }}^{\beta}$ (once recast on $[0,1]$, path constraints omitted by virtue of (I1))

$$
\begin{aligned}
& 1 / 2|h(x(1))|^{2} \rightarrow \min \\
& \dot{x}=\beta f(\beta t, x, u), t \in[0,1] \\
& x(0)=x^{0} \\
& |u| \leq T_{\max }
\end{aligned}
$$

with $f(t, x, u)=f_{0}(x)+B(x) u / m(t), B=\left[f_{1} f_{2}\right]$. In order to apply a Newton algorithm to find the first zero of $\phi$, we need $C^{1}$ regularity (Prop. 4.3 only gives Lipschitz continuity). This is achieved using the sensitivity analysis techniques of $[12,13]$. The idea is to construct an extremal family and to ensure (local) optimality by second order sufficient conditions. Let then $\beta_{0}$ be a parameter in $] 0, \bar{\beta}\left[\right.$, and let $\left(x_{0}, u_{0}\right) \in W_{n}^{1, \infty}([0,1]) \times L_{m}^{\infty}([0,1])$ be a solution to $(S P)_{T_{\max }}^{\beta_{0}}$. As before, the first order necessary condition holds in qualified form, and there is an absolutely continuous adjoint state $p_{0}$ such that (minimization of Hamiltonian)

$$
u_{0}=-T_{\max }{ }^{t}\left(p_{0} B\left(x_{0}\right)\right) /\left|p_{0} B\left(x_{0}\right)\right|
$$

since the Hamiltonian is $H(t, y, u, \beta)=\beta p\left(f_{0}(x)+B(x) u / m(t)\right)$. Of course, (5.1) is valid only when $p_{0} B\left(x_{0}\right)$ does not vanish, that is outside switching points again. By the same argument as in Section 3, we can prove that there are finitely many such points. Moreover, in accordance with Corollary 3.7, the numerical results at the end of the section will show that, in practice, there is at most one switching on the solution of the initial problem $(S P)_{T_{\max }}$. Nevertheless, we need to assume more here, namely that for any parameter $\beta$ in $] 0, \bar{\beta}[$, $(S P)_{T_{\max }}^{\beta}$ has an optimal control such that

(I2) $u(., \beta)$ is continuous.

Then, $u_{0}$ is even smooth and $y_{0}=\left(x_{0}, p_{0}\right)$ is solution of the two-point boundary value problem $(B V P)_{\beta_{0}}$ defined by the Hamilton equations (plus transversality)

$$
\begin{aligned}
\dot{x} & =\partial_{p} H\left(t, x, p, u(x, p), \beta_{0}\right) \\
\dot{p} & =-\partial_{x} H\left(t, x, p, u(x, p), \beta_{0}\right) \\
x(0) & =x^{0}, p(1)={ }^{t} h(x(1)) h^{\prime}(x(1))
\end{aligned}
$$

with $u(x, p)=-T_{\max }{ }^{t}(p B(x)) /|p B(x)|$. As a result, one can find an open neighbourhood $V$ of $\left(p_{0}(0), \beta_{0}\right)$ such that the maximal flow $\varphi(t, y, \beta)$ of $\dot{y}=\xi(t, y, \beta)$ - with $\xi$ the vector field defined by the right-hand side 
of $(5.2,5.3), \xi(t, y, \beta)=\left(\partial_{p} H(t, y, u(y), \beta),-\partial_{x} H(t, y, u(y), \beta)\right)$ - is defined on an open subset containing $[0,1] \times$ $\left\{x^{0}\right\} \times V$. On this neighbourhood, $(B V P)_{\beta}$ is equivalent to the shooting equation: find a covector $p^{0}$ such that

$$
S\left(p^{0}, \beta\right)=0
$$

with $S\left(p^{0}, \beta\right)=b\left(\varphi\left(1, x^{0}, p^{0}, \beta\right)\right)$ (and where $b(y)=p-{ }^{t} h(x) h^{\prime}(x)$ is the boundary condition corresponding to the second part in (5.4)). Finally, under the last two assumptions that, for any $\beta$ in $] 0, \bar{\beta}[$,

(I3) $\partial_{p} S(p(0, \beta), \beta)$ belongs to $G L_{n}(\mathbf{R})$;

(I4) the symmetric Riccati equation $(5.5,5.6)$ below has a bounded solution on $[0,1]$ (compare $[12,13])$

$$
\begin{aligned}
& \dot{Q}=-Q \mathcal{A}(t, \beta)-{ }^{t} \mathcal{A}(t, \beta) Q+Q \mathcal{B}(t, \beta) Q-\mathcal{C}(t, \beta) \\
& \left(\left(R^{f}-Q(1)\right) v \mid v\right) \geq 0, v \in \mathbf{R}^{4} \\
& \mathcal{A}(t, \beta)=\partial_{x} \xi_{1}(t, y(t, \beta), \beta) \\
& \mathcal{B}(t, \beta)=\partial_{p} \xi_{1}(t, y(t, \beta), \beta) \\
& \mathcal{C}(t, \beta)=\partial_{x} \xi_{2}(t, y(t, \beta), \beta) \\
& R^{f}=\left[\begin{array}{cc}
\mathbf{I}_{3} & 0 \\
0 & 0
\end{array}\right]\left(\mathbf{I}_{3} \text { identity matrix of order } 3\right)
\end{aligned}
$$

we have continuous differentiability and we are able to give the derivative of $\phi$ in closed form:

Proposition 5.1. Under assumptions (I1-I4), $\phi$ is $C^{1}$ on $] 0, \bar{\beta}\left[\right.$ and $\phi^{\prime}(\beta)=H(1, \beta) / \beta$.

For a detailed numerical verification of assumptions (I3, I4) in the constant mass case, see [5]. Here, we extend the explicit formula for $\phi^{\prime}$ to the more realistic variable mass case. We will use the following fact: if $W(\beta)$ is the value function of the abstract parametric optimization problem $(\widetilde{O})_{\beta}$ (parameter $\beta$ in a Banach space $\mathcal{B}$ ) with equality constraints

$$
\begin{aligned}
& J(z, \beta) \rightarrow \min \\
& F(z, \beta)=0
\end{aligned}
$$

with $J: \mathcal{Z} \times \mathcal{B} \rightarrow \mathbf{R}$ and $F: \mathcal{Z} \times \mathcal{B} \rightarrow \mathcal{Y}$ differentiable, $\mathcal{Z}, \mathcal{B}$ and $\mathcal{Y}$ Banach spaces, if for any $\beta$ in an open neighbourhood $V$ of $\beta_{0}$ the problem admits a solution $z(\beta)$ together with a multiplier $\lambda(\beta)$ in $\mathcal{Y}^{\prime}$ such that (KKT) conditions hold (in qualified form)

$$
\begin{aligned}
\partial_{z} L(z(\beta), \lambda(\beta), \beta) & =0, \partial_{\lambda} L(z(\beta), \lambda(\beta), \beta)=0 \\
L(z, \lambda, \beta) & =J(z, \beta)+\langle\lambda, F(z, \beta)\rangle_{\mathcal{Y}^{\prime}, \mathcal{Y}}
\end{aligned}
$$

and such that the mappings $\beta \mapsto z(\beta)$ and $\beta \mapsto \lambda(\beta)$ are differentiable, $W$ is differentiable and one has by reverse differentiation [4]:

$$
W^{\prime}(\beta)=\partial_{\beta} L(z(\beta), \lambda(\beta), \beta)
$$

Proof of Proposition 5.1. Thanks to assumption (I2), the control constraint is everywhere active in order that the parametric problem $(S P)_{T_{\max }}^{\beta}$ becomes a particular case of $(\widetilde{O})_{\beta}$, as one can see by setting $z=(x, u) \in \mathcal{Z}$ together with

$$
\begin{aligned}
& \mathcal{Z}=\mathcal{X} \times \mathcal{U}, \mathcal{X}=W_{n}^{1, \infty}([0,1]), \mathcal{U}=L_{m}^{\infty}([0,1]) \\
& \mathcal{Y}=L_{n}^{\infty}([0,1]) \times \mathbf{R}^{n} \times L^{\infty}([0,1]) \\
& J(z, \beta)=\frac{1}{2}|h(x(1))|^{2}, F(z, \beta)=\left[\begin{array}{l}
\dot{x}-\beta f(\beta t, x, u) \\
x(0)-x^{0} \\
1 / 2\left(|u|^{2}-T_{\max }^{2}\right)
\end{array}\right]
\end{aligned}
$$


Hence, we just need to check the assumptions of the sensitivity result of [13] on $(S P)_{T_{\max }}^{\beta}$ to apply (5.8). Let then $\beta_{0}$ be an arbitrary point in $] 0, \bar{\beta}\left[.(S P)_{T_{\max }}^{\beta_{0}}\right.$ has a solution $\left(x_{0}, u_{0}\right)$, together with an absolutely continuous adjoint state $p_{0}$ by virtue of (I1). The control $u_{0}$ defined by (5.1) is smooth (Assump. (I2)), and if $\widehat{H}$ is the augmented Hamiltonian

$$
\widehat{H}(t, x, p, u, \mu, \beta)=\beta p\left(f_{0}(x)+B(x) u / m(t)\right)+1 / 2 \mu\left(|u|^{2}-T_{\max }^{2}\right)
$$

with $\mu$ scalar multiplier associated with the inequality control constraint $1 / 2\left(|u|^{2}-T_{\max }^{2}\right) \leq 0$, one has $\nabla_{u} \widehat{H}\left(t, x_{0}, p_{0}, u_{0}, \mu_{0}, \beta_{0}\right)=0$ with

$$
\mu_{0}=\beta_{0}\left|p_{0} B\left(x_{0}\right)\right| /\left(m(t) T_{\max }\right) \geq 0
$$

As a result, $\mu_{0}$ is smooth and (I2) implies that strict complementarity holds: $\mu_{0}>0$ on $[0,1]$. Furthermore,

$$
\nabla_{u u}^{2} \widehat{H}\left(t, x_{0}, p_{0}, u_{0}, \mu_{0}, \beta_{0}\right)=\mu_{0} \mathbf{I}_{2}\left(\mathbf{I}_{2} \text { identity matrix of order } 2\right)
$$

is positive definite on $[0,1]$ and the strict Legendre-Clebsch condition is also fulfilled. Finally, with (I3) and (I4), all the assumptions required for the sensitivity analysis are valid, and there is an open neighbourhood of $\beta_{0}$ such that the mappings $\beta \mapsto z(\beta)$ and $\beta \mapsto \lambda(\beta)$ are defined and differentiable, with

$$
\lambda(\beta)=(-p(., \beta), p(0, \beta), \mu(., \beta)) \in W_{n}^{1, \infty}([0,1]) \times\left(\mathbf{R}^{n}\right)^{*} \times L^{\infty}([0,1])
$$

so that (KKT) conditions in the form (5.7) hold. Consequently, $\phi$ is differentiable in this neighbourhood, and

$$
\begin{aligned}
\phi^{\prime}(\beta) & =\lambda(\beta) \partial_{\beta} F(z(\beta), \beta)=\left\langle p(., \beta), f(\beta t, x(., \beta), u(., \beta))+\beta t \partial_{t} f(\beta t, x(., \beta), u(., \beta))\right\rangle_{\left(L^{\infty}\right)^{\prime}, L^{\infty}} \\
& =\int_{0}^{1} \partial_{\beta} H(t, \beta) \mathrm{d} t
\end{aligned}
$$

thanks to the regularity of the adjoint state $\left(p(., \beta) \in W_{n}^{1, \infty}([0,1]) \subset L_{n}^{2}([0,1]) \subset\left(L_{n}^{\infty}([0,1])\right)^{\prime}\right)$. Now, along the optimal trajectory for $\beta$,

$$
\begin{aligned}
d / d t(t H) & =H+t \dot{H} \\
& =H+t \partial_{t} H \\
& =\beta \partial_{\beta} H
\end{aligned}
$$

so that $\phi^{\prime}(\beta)=H(1, \beta) / \beta$. Since this is true in the neighbourhood of any point of $] 0, \bar{\beta}[$, the result holds on the whole open interval.

The numerical resolution is threefold. At the higher level, we use a continuation technique [3] on the maximum thrust: if $T_{\max }^{c}$ is the current thrust, if $\bar{\beta}^{c}$ is the first root of $\phi$ - where $\phi(\beta)$ is the value function of $(S P)_{T_{\max }^{c}}^{\beta}-$, the value $\bar{\beta}^{c}$ is used to initialize the resolution for the next thrust $T_{\max }^{+}$(of course, $T_{\max }^{+}<T_{\max }^{c}$ and $\bar{\beta}^{+}>\bar{\beta}^{c}$ ). For a given $T_{\max },(S P)_{T_{\max }}$ is solved by seeking the first root of $\phi$ (parametric approach of Sect. 4), taking advantage of the closed expression of $\phi^{\prime}$ provided by Proposition 5.1 for the Newton search. At the inner level, the evaluation of each $\phi(\beta)$ is performed by single shooting on the auxiliary problem $(S P)_{T_{\max }}^{\beta}$. The numerical 
values for the boundary conditions in (1.9) and (4.8) are:

$$
\begin{array}{ll}
P^{0}=11625 \mathrm{~km} & P^{f}=42165 \mathrm{~km} \\
e_{x}^{0}=0.75 & e_{x}^{f}=0 \\
e_{y}^{0}=0 & e_{y}^{f}=0 \\
L^{0}=\pi \mathrm{rad} & \delta=0.05112 \mathrm{~km}^{-1} \mathrm{~s} \\
m^{0}=1500 \mathrm{~kg} & \mu^{0}=398600.47 \mathrm{~km}^{3} \mathrm{~s}^{-2} .
\end{array}
$$

The optimal times computed by the method are given in Table 1 . They are about $5 \%$ smaller than those given by the constant mass model (compare [5]). The use of the orbital coordinates, much more stable numerically than the cartesian ones (the former arise as integrals of the unperturbed motion), makes the computation possible down to 0.3 Newton.

TABle 1. Optimal times for the coplanar transfer (variable mass model).

\begin{tabular}{|c|c||c|c|}
\hline$T_{\max }$ (Newtons) & $t_{f}$ (Hours) & $T_{\max }$ (Newtons) & $t_{f}$ (Hours) \\
\hline 60 & 14.732 & 2 & 420.10 \\
24 & 34.133 & 1.4 & 597.92 \\
12 & 69.294 & 1 & 839.97 \\
9 & 93.187 & 0.7 & 1195.7 \\
6 & 141.64 & 0.5 & 1685.2 \\
3 & 278.98 & 0.3 & 2838.4 \\
\hline
\end{tabular}

Since the value function $\phi$ is evaluated by single shooting on $(S P)_{T_{\max }}^{\beta}$, we may happen to find a local minimum of this auxiliary problem. Actually, we possibly only have an upper estimation of $\phi$, and the root $\bar{\beta}$ provided by the method may be not the first but just a local minimum of the original problem $(S P)_{T_{\max }}$. Nevertheless, there is still a fundamental difference with straightforward single shooting that does not treat the performance index value specifically: the Newton steps on the equation $\phi(\beta)=0$ provide an ordered search on the cost functional, so that too coarse local minima are avoided. Moreover, the method proves to be more robust numerically than the shooting procedure since it is less sensitive to the initializations of both the optimal time and the adjoint state. Indeed, thanks to the additional level of iteration above shooting, unaccurate shooting resolutions are permitted during the first Newton steps. Then, although the evaluation of $\phi$ gets distorted, the approximation available turns to be sufficient to initialize convergence. As the iterates get closer to the zero, the auxiliary shooting steps are more and more precise, and the desired accuracy is achieved at the solution. The optimal controls and trajectories for 60,3 and 0.3 Newtons are given in Figures 5.1 and 5.2.

Concerning switchings, for low thrusts (v.g. 3 and 0.3 Newtons) the controls present rapid variations which could be switchings. Now, the numerical experiments show that all these strong variations happen to be precisely located at the perigee (see Figs. 5.1 and 5.2): as a consequence, because of the previous study (Cor. 3.7), at most three of them can actually be switchings (variable mass case). Indeed, whatever the thrust, we can check numerically that there is always one and only one point (located at the perigee) where $\psi$ is numerically very close to zero, in accordance with Section 3 results. Since this evaluation of $\psi$ comes from an approximate computation of the optimal trajectory, it is not possible to decide whether there is or not a switching. Nevertheless, this observation makes us believe that, in practice, either there is no switching at all, or exactly one located at perigee.

\section{Conclusion}

We have reviewed the main geometric properties of the coplanar transfer of a satellite towards a geostationnary orbit, namely controllability and structure of optimal controls. We have also studied the convergence properties of the parametric method used to solve the control problem numerically. Among the coordinates available to 

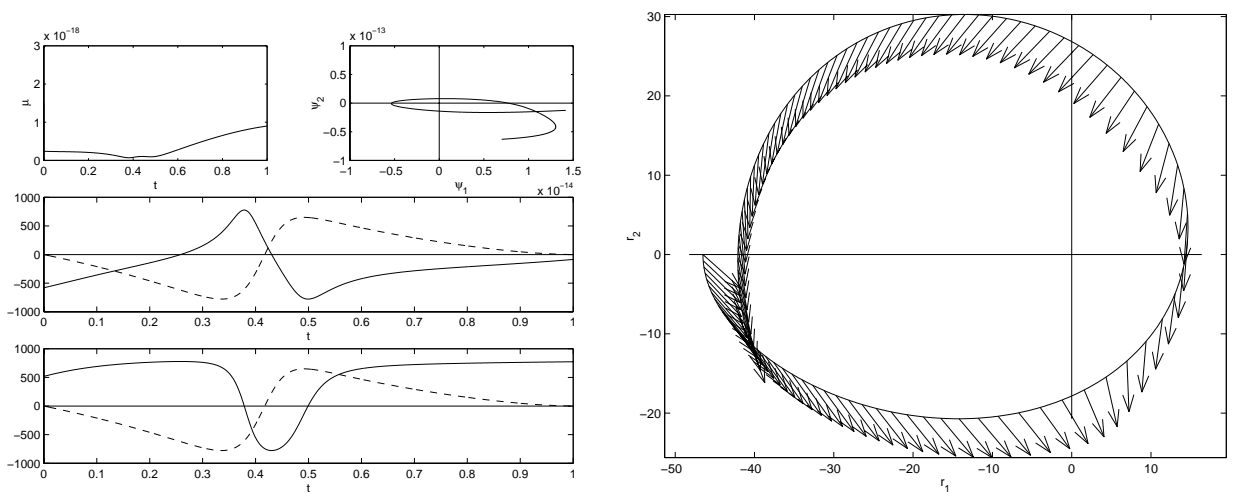

Thrust of 60 Newtons
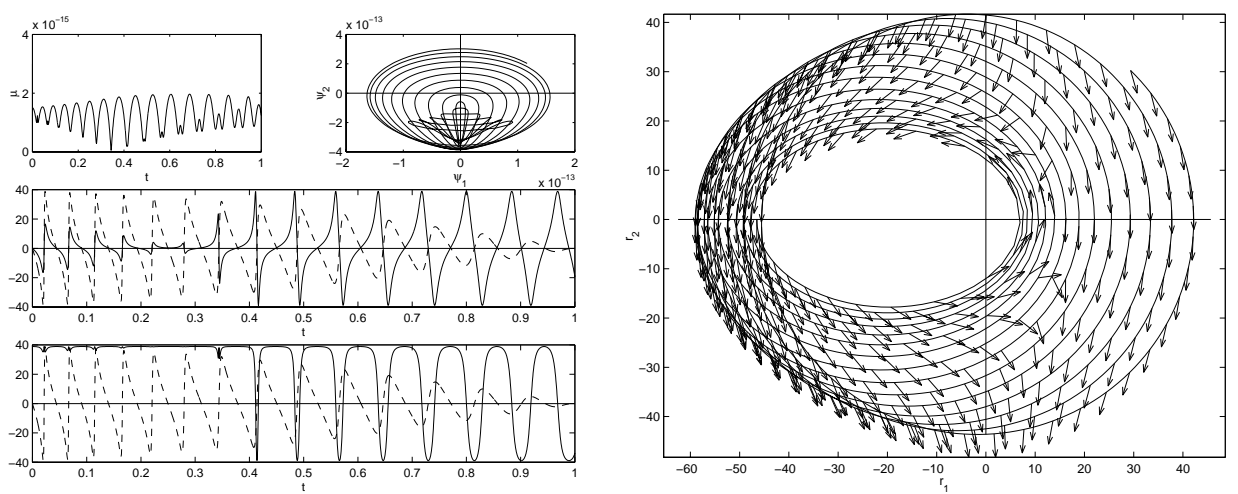

Thrust of 3 Newtons

FIGURE 5.1. For 60 and 3 Newtons, the trajectories are given on the right (the arrows represent the control). On the left, for both thrusts the two top graphs represent respectively $\mu$, Lagrange multiplier associated with the constraint on the modulus of the control, and $\psi$, the switching function. The other two graphs are the components of the control (solid line), $u_{1}$ and $u_{2}$, plus the plot of $e_{x} \sin L-e_{y} \cos L$ (dashed line) which vanishes at the perigee or the apogee. Whereas for 60 Newtons (strong thrust) the control is everywhere smooth, for 3 Newtons (low thrust) one can observe that at the point $\bar{t} \simeq 0.35$ where $\psi$ is almost passing through the origin, the satellite is exactly at the perigee. Besides, this point is situated at the change of phase (end of the semi-latus rectum increase, beginning of the eccentricity correction).

describe the mass varying system, the orbital parameters have proved to be the good choice of coordinates [16] since they provide not only the natural geometric framework to investigate the structure of the control, but also a numerically relevant change of variables. A challenging extension of this study would be to work on even more realistic hybrid (3D) models, taking into account additional continuous path and control constraints (eclipse phenomenons [7], etc.), together with logical constraints (v.g. satellites with several engines working in exclusive mode).

\section{REFERENCES}

[1] B. Bonnard and J. de Morant, Towards a geometric theory in the time minimal control of chemical batch reactors. SIAM J. Control Optim. 33 (1995) 1279-1311.

[2] B. Bonnard and G. Launay, Time minimal control of batch reactors. ESAIM: COCV 3 (1998) 407-467.

[3] J.B. Caillau, Contribution à l'étude du contrôle en temps minimal des transferts orbitaux. Ph.D. Thesis, ENSEEIHT, Institut National Polytechnique de Toulouse, France (2000). 

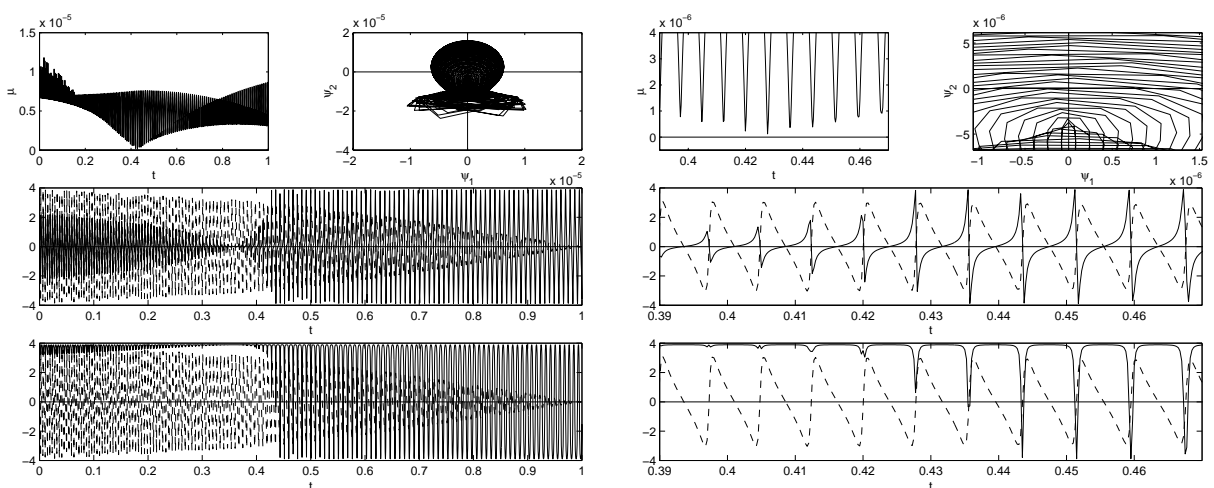

Thrust of 0.3 Newton

(detail)

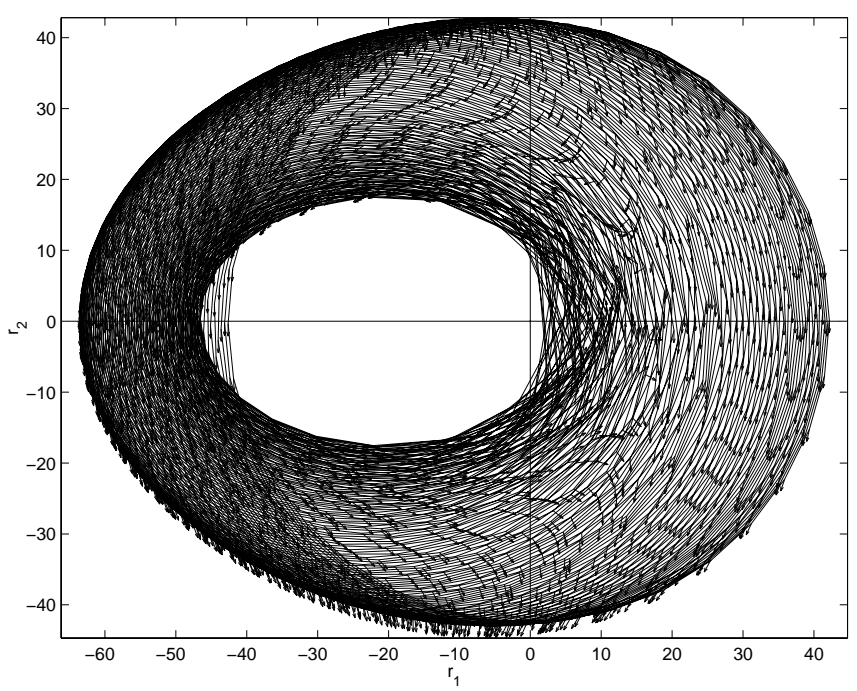

FiguRE 5.2. The picture for 0.3 Newtons (very low thrust) is the same as for 3 Newtons: all the rapid variations of the control are located at the perigee and there is only one of these points where $\psi$ almost vanishes, corresponding again to the change of phase. Here, there are more than 140 revolutions around the Earth.

[4] J.B. Caillau and J. Noailles, Continuous optimal control sensitivity analysis with AD, in Proc. of the 3rd International Conference on Automatic Differentiation. INRIA Nice, France (2000).

[5] J.B. Caillau and J. Noailles, Sensitivity analysis for time optimal orbit transfer. Optimization 49 (2001) 327-350.

[6] L. Cesari, Optimization Theory and Applications. Springer-Verlag (1983).

[7] C. Ferrier and R. Epenoy, Optimal control for engines with electro-ionic propulsion under constraint of eclipse. Acta Astronautica (to appear).

[8] M. Fliess, Variations sur la notion de contrôlabilité, in Quelques aspects de la théorie du contrôle. Journée Annuelle de la Société Mathématique de France (2000).

[9] S. Geffroy, R. Epenoy and J. Noailles, Averaging techniques in optimal control for orbital low-thrust transfers and rendez-vous computation, in $11^{\text {th }}$ International Astrodynamics Symposium. Gifu, Japan (1996) 166-171.

[10] M. Godbillon, Géométrie différentielle et mécanique analytique. Hermann, Paris (1985).

[11] V. Jurdjevic, Geometric control theory. Cambridge University Press (1997).

[12] K. Malanowski, Sufficient optimality conditions for optimal control subject to state constraints. SIAM J. Control Optim. 35 (1997) 205-227.

[13] K. Malanowski and H. Maurer, Sensitivity analysis for parametric optimal control problems with control-state constraints. Comp. Optim. Appl. 5 (1996) 253-283. 
[14] J. Noailles and J. Gergaud, A new method for the time optimal control problem and its application to low thrust orbital transfer. Workshop on low thrust transfers, Toulouse, France, French Space Agency, CNES (2000).

[15] J. Noailles and T.C. Le, Contrôle en temps minimal et transfert orbital à faible poussée. Équations aux dérivées partielles et applications, articles in honour of J.L. Lions for his $70^{\text {th }}$ birthday. Gauthier-Villars (1998) 705-724.

[16] H.J. Sussmann, Geometry and Optimal Control, in Mathematical Control Theory, Dedicated to Roger W. Brockett on his $60^{\text {th }}$ birthday, edited by J. Baillieul and J.C. Willems. Springer-Verlag (1998).

[17] H.J. Sussmann, Résultats récents sur les courbes optimales, in Quelques aspects de la théorie du contrôle. Journée Annuelle de la Société Mathématique de France (2000).

[18] O. Zarrouati, Trajectoires spatiales. CNES-Cepadues, Toulouse, France (1987). 